\title{
Risk Perception and Emotional Coping: A Pathway for Behavioural Addiction?
}

\author{
Ansgar Rougemont-Bücking Jeremy Grivel \\ Addiction Unit, Community Psychiatry Service, Department of Psychiatry, Centre Hospitalier Universitaire Vaudois \\ and University of Lausanne, Lausanne, Switzerland
}

\section{Key Words}

Dual-task interference $\cdot$ Addiction - Risk seeking $\cdot$ Working memory $\cdot$ Coping $\cdot$ EMDR $\cdot$ Implicit learning

\begin{abstract}
This article summarizes current concepts of the working memory with regard to its role within emotional coping strategies. In particular, it focuses on the fact that the limited capacity of the working memory to process now-relevant information can be turned into an advantage, when the individual is occupied by dealing with unpleasant emotion. Based on a phenomenon known as dual-task interference (DTI), this emotion can be chased by intense arousal due to clearly identifiable external stressors. Thus, risk perception might be used as a 'DTI inductor' that allows avoidance of unpleasant emotion. Successful mastery of risk adds a highly relevant dopaminergic component to the overall experience. The resulting mechanism of implicit learning may contribute to the development of a behavioural addiction. Besides its putative effects in the development of a behavioural addiction, the use of DTI might be of a more general interest for the clinical practice, especially in the field of psychotherapy.

(c) 2013 S. Karger AG, Basel
\end{abstract}

() 2013 S. Karger AG, Basel

$1022-6877 / 14 / 0202-0049 \$ 39.50 / 0$

\section{Introduction}

There is no perception emerging in a human being's brain that will receive more attention than the perception of an imminent threat to its life.

Human beings received throughout the evolutionary process a highly efficient and complex armament of adaptive mechanisms allowing them to cope with extreme stress and danger. In developed countries people are only very seldom and under exceptional conditions confronted with real and imminent threats to their physical integrity, e.g. like being exposed to a predator. However, concrete threats have been replaced by a constant concern about possible dangers to our psychological integrity and our social well-being. Yet, there are still some situations that directly threaten our physical integrity, for example exposure to considerable height with a risk of falling. It is of interest to note that human beings who generally strive to live under the most secure conditions, still have a strong tendency to develop individual ways of risk seeking in order to experience various levels of risk-related psychovegetative arousal [1]. A large amount of scientific literature is available about the psychological phenomena of risk and sensation seeking $[2,3]$. This article focuses on the effects of risk-related arousal on the attentional resources of the brain. The effect that risk perception imposes a rad-

\section{KARGER}

E-Mail karger@karger.com www.karger.com/ear
Ansgar Rougemont-Bücking

Addiction Unit, Community Psychiatry Service, Department of Psychiatry

Centre Hospitalier Universitaire Vaudois and University of Lausanne

7, rue St. Martin, $\mathrm{CH}-1003$ Lausanne (Switzerland)

E-Mail ansgar.rougemont-buecking@ chuv.ch 
ical switch in the processing of information having less vital importance may be conceptualized as a strategy to cope efficiently with unpleasant emotion.

According to current models of emotional coping, unpleasant emotion can be handled by three distinct mechanisms: firstly, resolving the underlying causes that provoke the unpleasant emotion; secondly, avoidance of entering into the unpleasant emotion, and finally, replacing the unpleasant emotion by a neutral or even pleasant emotion. The third option is of particular interest in concepts that elucidate the development and maintenance of a substance use disorder [4]. In these concepts the switching from an unpleasant condition towards a less unpleasant, neutral (or even pleasant) condition is achieved chemically by the consumption of a psychoactive substance. In this article the authors describe the pathways through which this switching between emotional conditions is obtained by a behavioural intervention. A special focus will be put on situations in which the shifting of emotional states is most abrupt and that have the highest impact on the brain's attentional resources. The situations that are of most relevance in developed countries are exposure to risk due to velocity and verticality.

\section{Height Perception as a Phylogenetically Determined Stressor}

'One of man's strongest fears is the fear of high places and falling'. This sentence introduces the monograph of Walk and Gibson [5] who developed the 'visual cliff paradigm which was used in a large amount of animal and human studies in order to investigate the effects of perception, locomotor control, developmental age and many other parameters on the expression of fear and avoidance of height [for a review, see 6]. There is strong evidence that fear of heights is largely determined through the evolutionary heritage and not acquired through associative learning [7]. However, it is still largely unknown in which manner the individual's response towards height stimuli adapts over time. Individual differences of sensitivity to heights might be best explained by some variability in the information-processing pathways between the sensory subsystems on the one hand, and the cognitive-executive functions on the other hand [8]. The influence of visual and postural perception along with the individual's ability to discriminate and interpret bodily sensations have been well documented as contributing factors for shaping the individual's responses to height stimuli $[9,10]$. There is some variability in the experiencing of the psychoveg- etative arousal due to the perception of height, as well as in the cognitive and emotional appraisal of this arousal. The matching between the sensory components related to height perception and arousal, on the one side, and the cognitive-discriminatory components of the appraisal, on the other side, contribute to the various manifestations of height tolerance or intolerance. Hence, individual reactions to heights can be differentiated as acrophobia, height intolerance, height tolerance, and height-seeking behaviour.

According to Lazarus' transactional model of stress [11], a potentially stressful event does not provoke a stress reaction unless it is appraised as threatening. In the case of height perception, the psychophysiological arousal is particularly intense. As this reactivity is phylogenetically determined for obvious reasons, no human being can remain unaffected in a situation of evident height exposure. It is supposed that both height-intolerant and heightseeking individuals have an increased susceptibility towards height-related bodily sensations. In the former group this susceptibility is appraised as emotionally unpleasant, and hence avoided, whereas in the latter group, the bodily experiencing of height is appraised as thrilling and exciting [12]. Most human beings are supposedly in the middle of this continuum between height intolerance and height seeking which places them into a height susceptibility that can be experienced by means of playful exploration. An enormous industry offers a large variety of products that aim to deliver 'verticality and/or velocity thrills' and millions of people spend a considerable amount of time and money to engage in risk-related activities.

This 'artificial' and voluntary experiencing of theoretically life-threatening situations may therefore be conceptualized as an attempt to expose the stress-coping system to a context for which it was once designed.

\section{Working Memory, Dual-Task Interference, and Stress Coping}

Working memory represents the capacity of storing and treating of now-relevant information. Current models of the ongoing information-processing system of the brain differentiate four subsystems of working memory: the phonological loop for auditory information, the visuospatial sketchpad for visuospatial information, the episodic buffer that links information across domains to form integrated and chronologicaly ordered units of information, and the central executive which controls and 
coordinates the operation of the three subsystems as well as the resource allocation within the working memory capacity.

According to the 'bottleneck metaphor', the human brain has a limited capacity for treating information generated by external or internal sources [13]. When a person tries to perform two tasks at the same time, in general a slowing down of the performance can be observed. This slowing is called the psychological refractory period [14], which was observed within a vast variety of experimental settings. This psychological refractory period is at the origin of another phenomenon which is the dual-task interference (DTI) [15]. This means that human beings show severe interference when performing two or several even very simple tasks at the same time. The tasks that have the ability to interfere one with another are characterized by the fact that they require both attentional resources and a distinct processing of information. These two prerequisite conditions are met in the case of intense arousal due to a risky situation and therefore the fact to enter in such a situation can be conceptualized as some kind of 'DTI-seeking behaviour'. Normally DTI is considered to be a 'brake' on the system. However, in the model proposed here, we examine the question whether DTI may act as a negative reinforcement and as a consequence may initiate and maintain a process of implicit emotional learning. These mechanisms may then contribute to the development of psychopathological manifestations like a behavioural addiction on the one hand, but may also contribute to beneficial effects of DTI in psychotherapy.

The term stress outlines experiences that are psychologically or physiologically challenging or threatening, stimulate the release of stress hormones, increase the organism's arousal level and consume its attentional resources $[16,17]$. Arousal refers to the level of alertness or activation of the organism $[18,19]$. It has been shown that the level of arousal has an influence on information processing. The Yerkes-Dodson law [20] describes an inverted U-shape relationship between arousal and performance where performance on tasks requiring attention is best at some mid-level of arousal and decreases as arousal becomes too low or too high.

In 1959, Easterbrook [21] tested the impact of various arousal-inducing manipulations on dual-task performance. Such experiments typically required the subjects to perform two distinct tasks simultaneously under different levels of arousal. He demonstrated that under high arousal levels, one task becomes of secondary and the other one of primary importance. Performance on the sec-

Risk Perception, Emotional Coping and Behavioural Addiction ondary task declines whereas performance on the primary task remains unaffected and in some instances improved. He concluded that high level of arousal lead to a narrowing of attention toward a single task.

In stressful situations, coping with the stressor becomes the primary task and enters in concurrence for allocation of attentional resources with any secondary task. Stress-induced arousal thus leads to a narrowing of attention toward coping with the stressful situation and decreases the performance on the concurrent secondary task. The point is that under some precise circumstances, this concurrent secondary task performance deterioration can be turned as an advantage, i.e. if the secondary task is ruminations of negative thoughts.

In this case, according to generally approved models of implicit emotional learning, the high psychophysiological arousal associated with risk perception chasing rumination of negative thoughts that are not directly related to the coping with the present danger can be conceptualized as some form of highly effective DTI. Furthermore, as the exposure to the danger was initiated voluntarily and as it is followed in most of the cases by the pleasant sensation of mastery and relief, the overall process corresponds to a negative conditioning learning process. By repetition of this process, exposure to risky situations may become one of the favoured mechanisms of emotional coping of the person. Moreover, it is known that acute stress potentiates dopaminergic reward pathways in the brain [22] and that the attention induced by risk perception can enhance the potential learning signal of the situation. Altogether, it can open a pathway that leads to behavioural addiction.

This stress-related ability to cope efficiently with unpleasant emotion might also contribute to the observation that sensation seekers express less fear and anxiety and are less receptive to the occurrence of unpleasant consequences of their actions [23,24]. It is possible that the state of stress approach, the appraisal of stress as something thrilling and challenging, and the preference of 'feeling stressed', instead of 'feeling bored or bothered' which are features commonly found in sensation seekers, can be understood as a clinical correlate to corroborate the assumption that sensation seekers use sensation seeking as a way to distract themselves [25]. The many neurobiological specificities that are found in sensation seekers and that involve the stress response system (hippocampal glucocorticoid receptors, HPA axis) [26], as well as the mesolimbic dopaminergic system signalling reward and salience [27], along with the fact that the sensation seeking frequently co-occurs with an addictive disorder [28, 29] might be an indicator for a deeply anchored tendency 
to cope with emotional stress rather by behaviours focusing on the emotional state itself than focusing on the underlying problem.

Problem solution is a quite recent phenomenon of stress coping that only appeared in the evolutionary process with the emergence of mankind. The predominant coping strategies in most species are to attack or to avoid the stressor. Thus, the tendency to react either by behaviourally or chemically mediated avoidance, while keeping the stress coping capacities frequently and efficiently engaged, might be a still highly active remnant of ancient evolutionary heredity.

\section{Towards a Model of Cue-Induced Negative Reinforcement and Contextually Maintained Positive Reinforcement of Behavioural Addiction}

The aforementioned studies and concepts mainly pertain to neurocognitive mechanisms regulating resource allocation of the working memory as a reaction to coping with stressful stimuli. When in such a setting, the first task corresponds to an unpleasant state (e.g. being occupied with unpleasant thoughts) then it is likely that the occurring DTI which is the result of the emerging second task can be conceptualized as a negative reinforcement. The negative state (unpleasant thoughts) is progressively replaced by a less unpleasant state, which is dealing with a stressful challenging situation. As long as the stress is bearable, the person will experience a relatively high level of psychological comfort. Of course, if the stress further increases, the level of psychological comfort will tend to decrease until, finally, an unpleasant state where the stress exceeds the individual's stress-coping competencies is obtained.

In clinical practice it can be commonly observed that patients keep themselves 'busy' by dealing with various forms of daily life problems and stressors that pop up repeatedly, even though therapists spend a lot of time working on problem resolution and on anticipation of similar problems in the future. By keeping themselves busy in dealing with these concrete situations, patients may avoid awareness of unpleasant emotion which would emerge as soon as the 'objective' level of daily life stress decreases. In addition, therapists, who often know or feel that their patients carry a heavy burden of unresolved psychological conflicts or of trauma-related psychopathology and who consider that their contribution as mental health professionals is to address these problems, refrain from approaching these 'painful' parts as the patient asks: 'I am too stressed right now, can we talk about this another time?' Interestingly, there is no scientific literature confirming or negating this hypothesis of a 'stress-seeking' tendency in patients who might use 'stress seeking' as a form of avoidance. However, in the light of the literature and of current concepts of classical conditioning, the high prevalence of the state (or also the trait) of 'being stressed' in psychiatric patients can be understood by two mechanisms that are closely related: Avoidance and distraction. Both mechanisms work together to play a crucial role in negative reinforcement: On the one hand, DTI leads to distraction from the unpleasant first task by automated, non-voluntary neurobiological mechanisms. On the other hand, engaging voluntarily in the second task that later generates DTI can be considered to be an act of avoidance.

These effects of DTI and avoidance become more powerful, and - importantly - easier to discriminate, when the second task is not responding to a stressful situation, but being exposed to a clearly identified risk. Already during the approach phase, a considerable part of the attentional resources of the working memory will be allocated towards task 2 (for this reason the slope of the resource allocation is steeper in figure $1 \mathrm{~b}$ than in figure 1a). Once exposed to the risk, the remaining unpleasant thoughts (task 1) are instantly replaced by executing task 2: responding to the now-relevant risk. As in many cases the risk perception is associated with the presence of a distinct stimulus (exposure to verticality when starting to climb, sitting at a roulette table in a casino, etc.), this risk exposure corresponds to a cue-induced negative reinforcement. Immediately after entering the risky situation, the totality of mental resources is allocated to the handling of the risky situation. As long as the individual's genuine and acquired capacities allow efficient coping with the risky situation (and even to succeed within this situation by gaining altitude or money) the execution of task 2 will be associated with a high level of psychological comfort. This state of 'handling the risk' can be conceptualized as a contextual positive reinforcement: the individual will continue to respond to the many various stimuli related to task 2 (e.g. climbing or gambling), but the overall experience will be highly biased by the ongoing contextual perception that the whole situation is highly risky. On the neurobiological level, both identification of a risk-related cue stimulus and successfully coping and succeeding within a highly risky situation are associated with dopaminergic neurotransmission in the ventral striatum [30]. When difficulties and possibilities of harmful consequences increase within task 2 and when the indi- 

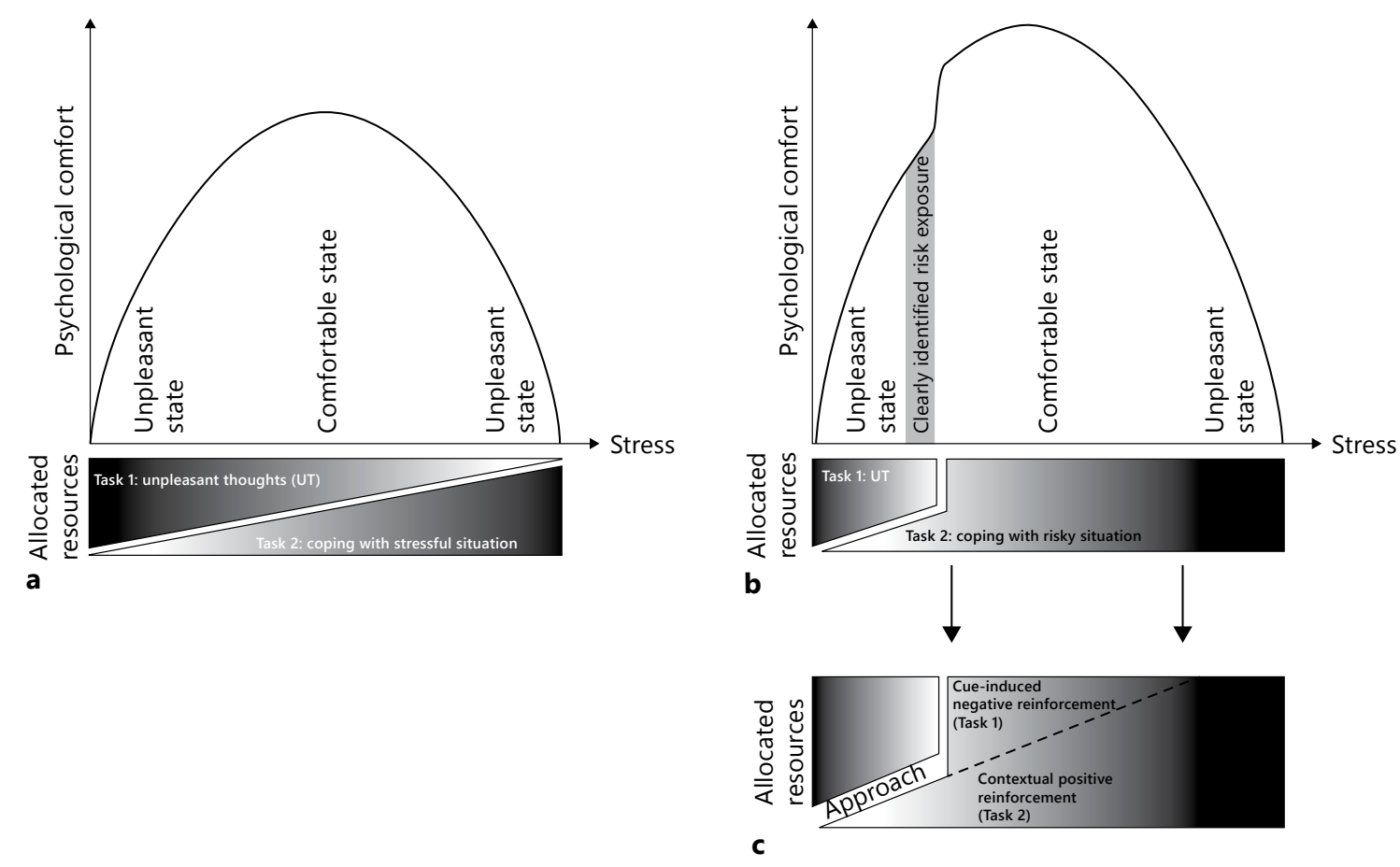

Fig. 1. a Progressive interference of a task (task 2, coping with stress) on another task (task 1, experiencing unpleasant thoughts). b Immediate interference on task 1 as soon as the individual gets exposed to a risk. c Components of cue-induced negative rein-

forcement as well as of a contextual positive reinforcement when the individual is approaching, then handling a risky situation, and eventually feeling overwhelmed by it. For further explanations, see text.

viduals coping competencies reach and cross their maximum capacities, then the individual will experience an unpleasant state of being overwhelmed in task 2. However, the price to pay to be in this state (e.g. feeling extremely stressed or fearful, getting hurt, losing money) may be 'less expensive' than the price that would have been to pay if the individual had stayed in task 1 (unpleasant thoughts). That means that the advantage of having performed a negative reinforcement through risk-related DTI outweighs the disadvantage of having experienced a state of unpleasant stress or objective harm when arriving at a very high level of stress within task 2 . The occurrence of an aversive consequence in task 2 is furthermore limited by the fact that risk-seeking users of task 2 are normally able to limit the stress level of task 2 , which means they will get out of the situation before harm objectively occurs. Therefore, aversive consequences that would have limiting effect on the overall implicit and associative learning process occur rather seldom. 'Risk-seeking users' will rather become quite competent and successful in handling high levels of risk-related stress, and they will

enjoy a maximum range of psychological comfort in doing so, without entering too often the high-end, unpleasant area of task 2.

\section{Discussion}

The problems of many psychopathological manifestations in the clinics are due to maladaptive learning processes in the field of emotional coping with stress. Human beings are constantly striving to maintain physical and psychological homeostasis within a world that constantly brings changing and frequently unexpected challenging events. Through the evolutionary process all living organisms are well equipped in order to deal with this neverending course of stressors. The many stress-related coping mechanisms oriented towards the outer world have been extensively studied in the animal model, as well as in humans. This research on stress- and anxiety-related disorders led to a plethora of literature in the field of classical and operant conditioning always referring to pro- 
cesses of implicit associative learning [31]. However, in addition to clinical manifestations oriented to the outer world (e.g. fearful avoidance, aggressive behaviour, flight, freeze, etc.), there may be many behavioural strategies which are primarily oriented to the inner world, the proper emotional coping system of the individual. A good example of the coexistence of behavioural mechanisms of emotional coping on both the external and the internal surface is obsessive-compulsive disorder. In obsessivecompulsive disorder, obsessions are mental actions (only identifiable through careful questioning) that have the function to reduce abnormal anxiety due to exaggerated beliefs or false convictions. The same dysfunctional beliefs can also provoke behavioural manifestations on the external surface, which then are called compulsions, and in analogy to obsessions have the role to decrease the level of anxiety.

The authors of this article propose that the phenomenon of DTI is one of these mechanisms of stress regulation at the internal level. As shown in the paragraphs before, DTI can contribute to dampen unpleasant emotion by forcing the attention of the central executive to be focused on a task of higher relevance, and being less disagreeable.

To date there are no studies, to our knowledge, allowing to confirm or to negate our hypothesis of a DTI component contributing in the development of behavioural addiction. Furthermore, interpretation of the existing data is not easy with regard to this hypothesis. In the field of pathological gambling, the behavioural addiction most investigated, there are some publications investigating the links between sensation or risk seeking and gambling [32-34]. The results are often inconsistent to answer the question whether and in which way sensation seeking and pathological gambling might be correlated. Furthermore, using these studies to test our hypothesis might be limited by the fact that the overall reactivity to many types of external stimuli and contexts was tested. There is very little data to to help understand in which way the internal processing and experiencing of unpleasant emotion is affected when engaging into gambling: Fortune and Goodie [32] showed that pathological gamblers score generally higher on the two subscales of the Zuckerman Sensation Seeking Scale that measure disinhibition and boredom susceptibility, and that they do not differ on the other two subscales assessing thrill and experience seeking, in comparison to healthy controls. This observation is supported by the study of Nower et al. [25] who demonstrated that male pathological gamblers were more likely than non-gambling peers to use avoidant stress-coping strategies, and to seek emotional stimulation in order to distract themselves. In sum, these data suggest that pathological gamblers who are highly sensitive to boredom and internal monotony or rumination of negative thoughts, are more likely to engage in activities leading to immediate stimulation and arousal due to actual behaviour and they are less likely to engage in behaviour aimed to prepare hypothetical behaviour in the future. Therefore, gambling may be used, along with other thrilling activities, as a DTI by a subgroup of the population that is characterized by features such as boredom susceptibility and avoidant coping styles in order to reach an optimum level of psychological homeostasis.

The finding that many persons have difficulty engaging in mental actions in order to obtain rather discreet modifications of their psychological state is very well known. It explains, in part, why psychotherapies that include components of mental practising (attentional respiration and 'body scanner' in mindfulness meditation, for example) are not suitable for all patients that normally 'should' benefit from these treatments. It is probable that the reactivity of human beings towards stimuli that are generated in the inner or the outer world (including pharmacological agents that shortcut effects on inner perception but are delivered from the outer world) can be considered as being distributed on a continuum. Some people are extremely sensitive to external stimulation and much less sensitive to experiencing or modulating internal processes by means of mental practice, whereas for other people the opposite might be true. A large number of persons are supposed to be in the middle of this continuum, having a quite well-developed sensitivity to stimuli coming from both internal and external sources and being able to modulate this experience by means of mental practice.

What gambling and rock climbing have in common is the fact that the person who is engaging in these activities will automatically feel aroused as the perception of risk and potential damage is highly present in these activities. These activities therefore represent 'DTI inductors' generated in a very reliable manner by the outer world. The popularity of 'verticality exposing' activities (climbing, bungee jumping, etc.), but also of other activities with risks related to velocity (most motor sports, downhill bicycling, etc.) or activities presenting both exposure to verticality and to velocity (paragliding, skiing, rollercoaster, etc.) might be explained by this concept. With regard to the arousal perceived while gambling, it is crucial to note that the arousal is highly dependent on the fact that the 
game is about 'real money' representing a real risk of damage and that the arousal is much less present in case of an only symbolic stake [35]. This fact hints to the presence of an acquired cognitive component which contributes to gambling-induced arousal, whereas the arousal due to verticality or velocity exposure is mainly due to the phylogenetical heritage and thus largely free of sociocultural learning influences.

The authors also suggest that the phenomenon of DTI may be instrumentalized and practised in specific forms of psychotherapy in order to strengthen the individual's stress-coping competencies. The potential benefit of DTI was shown in works by Holmes et al. [36]: they showed that a visuospatial task (playing the computer game 'Tetris') could inhibit the memory consolidation of traumatic material in individuals who had viewed a film with traumatic content $30 \mathrm{~min}$ prior to being assigned to the 'Tetris' task. Compared to the no-task condition, the 'Tetris' condition produced a significant reduction in flashback frequency over a 1-week observation period.

Another example of an intervention on the brain's information processing is EMDR (eye-movement desensitization and reprocessing). EMDR is an effective form of psychotherapy for patients suffering from PTSD [37]. Its neurobiological underpinnings are not yet fully understood but there is some evidence that EMDR works through interference on the working memory [38-41]. The most prominent feature of this therapy are the eyemovements that the patient has to perform (guided by the therapist) while re-experiencing a traumatic event. The simultaneous performance of two tasks (the recall of a traumatic event and the execution of bilaterally alternating horizontal eye-movements) can be considered as a 'therapeutical DTI setup'. It was shown that eye-movements interfere effectively with visuospatial memory that is activated during trauma imagination. As a consequence, the vividness and emotiveness of the traumatic images are reduced [42-44]. In general, eye-movements appear to be particularly efficacious for interfering with the working memory's simultaneous processing of another task also using the visuospatial sketchpad $[45,46]$.

These observations are consistent with the 'bottleneck' model of the working memory that posits that information belonging to the same modality of information (visuospatial or phonological) show a higher degree of DTI. To confirm this, a recent study comparing interference of acoustical tones with interference of eye-movements on the recollection of negative memories showed that eyemovements affect the working memory to a higher degree than acoustical tones [47].

Risk Perception, Emotional Coping and Behavioural Addiction
There also might be some analogy between the effects of eye-movements in EMDR and the effects of attentional breathing in mindfulness based cognitive therapy (MCBT) or in other forms of meditation or relaxation exercises. Attentional breathing in MBCT may be considered as DTI on the working memory. Thus, it was supposed that MCBT derives some of its beneficial effects from taxing working memory capacities during recall of negative ideation [48].

Another example for the clinical application of DTI can be found in the field of non-pharmacological treatments for chronic pain. Interventions that are based on the distraction of the attentional resources have been described at many times $[49,50]$, but these forms of therapy only found a limited degree of use in clinical practice. It is possible that most patients suffering from chronic pain have difficulties in actively practicing and performing these mental exercises [51]. Interestingly, use of EMDR for chronic pain patients showed quite promising results [52].

With regard to depression, distraction, especially when it contains an external component, is a validated therapeutical intervention to modify depressed mood [53-55].

The finding that DTI-containing therapies may not be suitable for all patients who present affections that could theoretically benefit from these interventions points to the question of how to increase the implementation, acceptation, and efficacy of DTI-related therapeutical interventions. To answer this question it seems to be of importance to note that the forms of DTI that seem most effective for obtaining negative reinforcement contain a well-defined discriminatory external element that induces DTI. It is probable that the presence of an outer world distractor facilitates the process of DTI, in contrast to a progressively increasing DTI, in which the secondary task is not associated by clearly identifiable stimuli (cue and contextual). Therefore, the induction and maintenance of DTI in EMDR by performing eye movements, guided by the therapist, or of DTI in mindfulness relaxation by performing attentional breathing, guided by the voice of the therapist, might be better accepted and more effective than DTI-containing therapeutical interventions that have no component of external guidance. In addition, DTI interventions should be in the zone of comfort in which both tasks are quite equally treated by the central executive. The shifting between these two tasks may contribute to a more adaptive treatment of information (related to task 1), according to the working memory hypothesis of EMDR, cited above. 
Of what use is our model of DTI for treating behavioural addictions? To our knowledge there are no studies that investigate the effects of distraction (or DTI) on behavioural addictions. However, some of the aforementioned treatment techniques that contain DTI components (such as mindfulness meditation, or EMDR) showed promising results in treating substance use disorders $[56,57]$. Especially in the domain of nicotine craving, where it was shown that attentional resources are highly biased towards smoking-related stimuli $[58,59]$, several studies suggest that interventions aiming to modify the attribution of attentional resources might be beneficial. Thus, it was shown that a concomitant attentional interference which was driven by auditory instructions showed to reduce significantly nicotine craving [60]. Similar results were obtained on cigarette craving when attentional breathing was used as an interfering task [61]. Kemps et al. [62] who explored appetitive reactivity in a large sense showed that a concurrent visuospatial task contributes to an effective reduction of craving for chocolate.

As DTI-related behavioural addiction was acquired within a process of negative reinforcement, it appears to be of paramount priority to focus the therapeutical effort on exploring the task 1 - the unpleasant condition. Therapists should accompany the patient by means of empathetic questioning in order to investigate the presence of hidden forms of anxiety and/or depression. Often, patients presenting addictive behaviours have considerable difficulties in acknowledging a psychological suffering, other than the suffering directly related to the behavioural excess (task 2) that pushed them to seek professional help. As the unpleasant emotions and thoughts of task 1 are contained by stress and risk-seeking behaviours of task 2 in order to stay at a low level of consciousness, any attempt to reduce or to abolish the availability or experiencing of task 2 will be perceived as a menace to the individual's psychological equilibrium. Thus, reducing task 2 without simultaneously reducing of the aversive components in task 1 will not be well tolerated by these patients. Reducing the aversive components in task 1 can be achieved by various forms of psychotherapy, coupled with pharmacological treatment in patients who are willing to accept therapeutical interventions on task 1 (instead of focusing mainly on task 2). As soon as the negative condition of task 1 is reduced, intervention on task 2 can take place. Intervention on task 2 has to focus on its two distinct components: DTIrelated effects, and dopaminergic effects of salience and reward.
When focusing on DTI-related effects it would be useful to obtain a DTI which is no longer driven by the identification of a highly relevant element of risk. Concretely, the patient, assisted by the therapists, should explore whether there are other, no-risk-related activities in which he can engage as an alternative task 2 and still divert himself effectively from task 1 .

There remains the problem of how to modify the dopaminergic regulation related to the highly thrilling task 2. Unfortunately, the therapeutical interventions known from pathological gambling that aim to reduce the reactivity towards cue and contextual stimuli that provoke arousal in a gambling situation [63] are not suitable for reducing natural risk-related arousal. These interventions are known as 'stimulus control', aiming to maintain executive control not to enter in a highly arousing situation (of gambling). The other well-known technique is 'imaginal desensitization' by which the psychovegetative reactivity towards contextual stimuli of gambling can be reduced. As said before, arousal related to verticality (or velocity) is mainly phylogenetically determined, and its function to prevent harm is still useful and important in daily life. Therefore, any intervention aiming to decrease this reactivity would put the patient at risk of reacting inadequately in the future.

In all cases of behavioural change, when finding and exploring alternative activities for task 2 , the reduction of risk and thrill exposure will be associated with a considerable decrease of dopamine activity in the ventral striatum. Typically, as in most cases of withdrawal from an addictive behaviour, this reduced dopamine reactivity manifests clinically as a motivational difficulty of the patient to stick to the process of change. In the cognitive-behavioural therapy setting, the role of the therapist is to support and accompany the patient in this process by defining gradually adapted versions of the alternative task or behaviour. In the long-term recovery from an addictive disorder, the addicted patient has to find and experience alternative ways of stimulating the dopaminergic pathways of the striatum. As said above, all this change with regard to task 2 can only be achieved when the therapist is able to show the patient a way of exploring gently and of reducing efficiently the unpleasant condition associated with task 1 . As long as no substantial improvement concerning the exploration and reduction of the unpleasant condition is achieved, the patient will be at risk of replacing one behavioural excess with another.

As the neurobiological underpinnings of the effects of DTI are becoming better and better understood, it might be important that both patients and mental healthcare
56

Eur Addict Res 2014;20:49-58 DOI: $10.1159 / 000353589$
Rougemont-Bücking/Grivel 
professionals become informed about these effects, and about the importance of using components of distraction and double task in their therapies. Till now, distraction or dual-task elements are generally considered to be odd, non-serious tools with a shamanic background. By explaining to a broader public the mechanisms and effects of distraction and dual-task in mental health, clinicians and researchers will be able to add another stone to the bridge being built between basic science and the clinics.

\section{References}

$>1$ Trimpop RM: Risk homeostasis theory: problems of the past and promises for the future. Saf Sci 1996;22:119-130.

-2 Zuckerman M, Kuhlman DM: Personality and risk-taking: common biosocial factors. J Pers 2000;68:999-1029.

$>3$ Cherpitel CJ: Substance use, injury, and risktaking dispositions in the general population. Alcohol Clin Exp Res 1999;23:121-126.

4 Wills TA, Hirky AE: Coping and substance abuse: a theoretical model and review of the evidence; in Zeidner M, Endler NS (eds): Handbook of Coping: Theory, Research, and Applications. New York, Wiley, 1996, pp 279-302.

$>5$ Walk RD, Gibson EJ: A comparative and analytical study of visual depth perception. Psychol Monogr 1961;75:1-44.

6 Adolph KE, Kretch KS: Infants on the edge: beyond the visual cliff; in Slater A, Quinn P (eds): Refreshing Developmental Psychology: Beyond the Classic Studies. London, SAGE, 2012.

$>7$ Poulton R, Davies S, Menzies RG, Langley JD, Silva PA: Evidence for a non-associative model of the acquisition of a fear of heights. Behav Res Ther 1998;36:537-544.

-8 Boffino CC, de Sa CS, Gorenstein C, Brown RG, Basile LF, Ramos RT: Fear of heights: cognitive performance and postural control. Eur Arch Psychiatry Clin Neurosci 2009;259:114-119.

$>9$ Coelho CM, Wallis G: Deconstructing acrophobia: physiological and psychological precursors to developing a fear of heights. Depress Anxiety 2010;27:864-870.

$>10$ Davey GC, Menzies R, Gallardo B: Height phobia and biases in the interpretation of bodily sensations: some links between acrophobia and agoraphobia. Behav Res Ther 1997;35:997-1001.

11 Lazarus R, Folkman S: Stress, Appraisal, and Coping. New York, Springer, 1984.

$>12$ Salassa JR, Zapala DA: Love and fear of heights: the pathophysiology and psychology of height imbalance. Wild Environ Med 2009; 20:378-382.

13 Pashler H: Dual-task interference in simple tasks: data and theory. Psychol Bull 1994;116: 220-244.

14 Telford CW: The refractory phase of voluntary and associative responses. J Exp Psychol 1931;14:1-36.

15 Navon D, Miller J: Role of outcome conflict in dual-task interference. J Exp Psychol Human 1987;13:435-448.
16 Sapolsky RM: Stress and cognition; in Gazzaniga MS (ed): The Cognitive Neuroscience. Cambridge, MIT Press, 2004, pp 1031-1042.

17 Eysenck MW: Attention and Arousal, Cognition and Performance. New York, Springer, 1982.

18 Duffy E: Activation and Behavior. New York, Wiley, 1962.

19 Humphreys MS, Revelle W: Personality, motivation, and performance: a theory of the relationship between individual differences and information processing. Psychol Rev 1984;91: 153-184.

20 Yerkes RM, Dodson JD: The relation of strength of stimulus to rapidity of habit formation. J Comp Neurol Psychol 1908;18:459482.

21 Easterbrook JA: The effect of emotion on cue utilization and the organization of behavior. Psychol Rev 1959;66:183-201.

22 Ungless MA, Argilli E, Bonci A: Effects of stress and aversion on dopamine neurons: implications for addiction. Neurosci Biobehav Rev 2010;35:151-156.

23 Gunnarsdottir ED, Pingitore RA, Spring BJ, Konopka LM, Crayton JW, Milo T, Shirazi P: Individual differences among cocaine users. Addict Behav 2000;25:641-652.

24 Blanchard MM, Mendelsohn D, Stamp JA: The HR/LR model: further evidence as an animal model of sensation seeking. Neurosci Biobehav Rev 2009;33:1145-1154.

25 Nower L, Derevensky JL, Gupta R: The relationship of impulsivity, sensation seeking, coping, and substance use in youth gamblers. Psychol Addict Behav 2004;18:49-55.

26 Kabbaj M: Neurobiological bases of individual differences in emotional and stress responsiveness: high responders-low responders model. Arch Neurol 2004;61:1009-1012.

27 Hooks MS, Jones GH, Smith AD, Neill DB, Justice JB Jr: Response to novelty predicts the locomotor and nucleus accumbens dopamine response to cocaine. Synapse 1991;9: 121-128.

28 Zuckerman M: Sensation seeking and the endogenous deficit theory of drug abuse. NIDA Res Monogr 1986;74:59-70.

$>29$ Franques P, Auriacombe M, Piquemal E, Verger M, Brisseau-Gimenez S, Grabot D, Tignol J: Sensation seeking as a common factor in opioid-dependent subjects and high-risk sport practicing subjects. A cross-sectional study. Drug Alcohol Depend 2003;69:121126.
30 Schultz W, Preuschoff K, Camerer C, Hsu M, Fiorillo CD, Tobler PN, Bossaerts P: Explicit neural signals reflecting reward uncertainty. Philos Trans R Soc Lond B Biol Sci 2008;363: 3801-3811.

-31 Mineka S, Oehlberg K: The relevance of recent developments in classical conditioning to understanding the etiology and maintenance of anxiety disorders. Acta Psychol 2008; 127:567-580.

32 Fortune E, Goodie A: The relationship between pathological gambling and sensation seeking: the role of subscale scores. J Gambl Stud 2010;26:331-346.

33 Cyders MA, Smith GT: Clarifying the role of personality dispositions in risk for increased gambling behavior. Pers Individ Dif 2008;45: 503-508.

34 Saez-Abad C, Bertolin-Guillen JM: Personality traits and disorders in pathological gamblers versus normal controls. J Addict Dis 2008;27:33-40.

$>35$ Anderson G, Brown RI: Real and laboratory gambling, sensation-seeking and arousal. Br J Psychol 1984;75:401-410.

36 Holmes EA, James EL, Coode-Bate T, Deeprose $\mathrm{C}$ : Can playing the computer game 'Tetris' reduce the build-up of flashbacks for trauma? A proposal from cognitive science. PLoS One 2009;4:e4153.

37 Bisson J, Andrew M: Psychological treatment of post-traumatic stress disorder (PTSD). Cochrane Database Syst Rev 2007; 3:CD003388

38 Bergmann U: EMDR's neurobiological mechanisms of action: a survey of 20 years of searching. J EMDR Pract Res 2010;4:22-42.

39 Gunter RW, Bodner GE: EMDR works. But how? Recent progress in the search for treatment mechanisms. J EMDR Pract Res 2009;3: 161-168.

40 Hofmann A, Galley N: Theorien zur Wirksamkeit von EMDR; in Hofmann A (ed): EMDR - Therapie psychotraumatischer Belastungssyndrome. Stuttgart, Thieme, 2004, vol 2, pp 72-76.

41 Maxfield L, Melnyk WT, Hayman GCA: A working memory explanation for the effects of eye movements in EMDR. J EMDR Pract Res 2008;2:247-261.

42 Andrade J, Kavanagh D, Baddeley A: Eyemovements and visual imagery: a working memory approach to the treatment of posttraumatic stress disorder. Br J Clin Psychol 1997;36:209-223. 
43 Kavanagh DJ, Freese S, Andrade J, May J: Effects of visuospatial tasks on desensitization to emotive memories. Br J Clin Psychol 2001; 40:267-280.

44 Lilley SA, Andrade J, Turpin G, Sabin-Farrell $\mathrm{R}$, Holmes EA: Visuospatial working memory interference with recollections of trauma. $\mathrm{Br} J$ Clin Psychol 2009;48:309-321.

45 Pearson D, Sahraie A: Oculomotor control and the maintenance of spatially and temporally distributed events in visuo-spatial working memory. Q J Exp Psychol A 2003;56: 1089-1111.

46 Kemps E, Tiggemann M: Reducing the vividness and emotional impact of distressing autobiographical memories: the importance of modality-specific interference. Memory 2007; 15:412-422.

-47 Van den Hout MA, Engelhard IM, Rijkeboer MM, Koekebakker J, Hornsveld H, Leer A, Toffolo MB, Akse N: EMDR: Eye movements superior to beeps in taxing working memory and reducing vividness of recollections. Behav Res Ther 2011;49:92-98.

48 Van den Hout MA, Engelhard IM, Beetsma D, Slofstra C, Hornsveld H, Houtveen J, Leer A: EMDR and mindfulness. Eye movements and attentional breathing tax working memory and reduce vividness and emotionality of aversive ideation. J Behav Ther Exp Psychiatry 2011;42:423-431.
49 Eccleston C: Chronic pain and distraction: an experimental investigation into the role of sustained and shifting attention in the processing of chronic persistent pain. Behav Res Ther 1995;33:391-405.

50 Eccleston C: The attentional control of pain: methodological and theoretical concerns. Pain 1995;63:3-10.

51 Lau OWY, Leung LNY, Wong LOL: Cognitive behavioural techniques for changing the coping skills of patients with chronic pain. Hong Kong J Occup Ther 2002;12:13-20.

52 Grant M, Threlfo C: EMDR in the treatment of chronic pain. J Clin Psychol 2002;58:15051520.

53 Morgan AJ, Jorm AF: Self-help interventions for depressive disorders and depressive symptoms: a systematic review. Ann Gen Psy 2008; 7:13.

54 Fennell MJ, Teasdale JD: Effects of distraction on thinking and affect in depressed patients. Br J Clin Psychol 1984;23:65-66.

55 Fennell MJV, Teasdale JD, Jones S, Damlé A: Distraction in neurotic and endogenous depression: an investigation of negative thinking in major depressive disorder. Psychol Med 1987;17:441-452.

56 Skanavi S, Laqueille X, Aubin HJ: Mindfulness based interventions for addictive disorders: a review (in French). Encephale 2011;37: 379-387.
57 Hase M, Schallmayer S, Sack M: EMDR reprocessing of the addiction memory: pretreatment, posttreatment, and 1-month follow-up. J EMDR Pract Res 2008;2:170-179.

58 Khazaal Y, Zullino D, Billieux J: The Geneva Smoking Pictures: development and preliminary validation. Eur Addict Res 2012;18:103109.

59 Vollstadt-Klein S, Loeber S, Winter S, Lemenager T, von der Goltz C, Dinter C, Koopmann A, Wied C, Winterer G, Kiefer F: Attention shift towards smoking cues relates to severity of dependence, smoking behavior and breath carbon monoxide. Eur Addict Res 2011;17:217-224.

-60 May J, Andrade J, Willoughby K, Brown C: An attentional control task reduces intrusive thoughts about smoking. Nicotine Tob Res 2012;14:472-478.

61 Shahab L, Sarkar BK, West R: The acute effects of yogic breathing exercises on craving and withdrawal symptoms in abstaining smokers. Psychopharmacology 2013;225:875-882.

62 Kemps E, Tiggemann M, Hart G: Chocolate cravings are susceptible to visuo-spatial interference. Eat Behav 2005;6:101-107.

63 Gooding P, Tarrier N: A systematic review and meta-analysis of cognitive-behavioural interventions to reduce problem gambling: hedging our bets? Behav Res Ther 2009;47: 592-607. 